\title{
Photocoagulation for juxtapapillary retinal hemangioma in a young girl: A case report
}

\author{
SATORU KASE and SUSUMU ISHIDA \\ Department of Ophthalmology, Faculty of Medicine and Graduate School of Medicine, \\ Hokkaido University, Sapporo, Hokkaido 060-8638, Japan
}

Received August 14, 2018; Accepted March 8, 2019

DOI: $10.3892 / \mathrm{mco} .2019 .1824$

\begin{abstract}
Hippel Lindau (VHL) disease is caused by inactivation of the VHL tumor suppressor gene, resulting in formation of multiple systemic tumors. Juxtapapillary retinal capillary hemangioma $(\mathrm{JRCH})$ is one of the major manifestations in VHL disease; however, treatments are challenging, especially in children. The present study reports the case of a 6-year-old girl with suspected VHL disease presenting with JRCH. Fluorescein angiography demonstrated marked dye leakage from the tumor. Retinal hemorrhage occurred around the tumor 7 months later. Laser photocoagulation of the tumor tissue was safely and successfully conducted without general anesthesia. The hemorrhage diminished after laser photocoagulation. Optical coherence tomography demonstrated mild resolution of an elevated lesion in JRCH with contraction of the vitreoretinal interface over the tumor. Laser photocoagulation may be considered the first-line treatment for young patients with JRCH, although the primary physicians must have the necessary skills and be able to establish good doctor-patient relationships, even with children.
\end{abstract}

\section{Introduction}

von Hippel Lindau (VHL) disease is caused by inactivation of the VHL tumor suppressor gene, and subsequent dysregulation of hypoxia-inducible factor expression, which leads to multiple tumors commonly arising in the retina, cerebellum, spinal cord, and kidney (1). Tumors with different histopathologies can spatio-temporally occur in various organs such as the central nervous system and pancreas (2). Retinal hemangiomas in patients with VHL disease are sight-threatening tumors in the young (3). If the tumors are localized in the peripheral

Correspondence to: Dr Satoru Kase, Department of Ophthalmology, Faculty of Medicine and Graduate School of Medicine, Hokkaido University, 15 Kita Street, Sapporo, Hokkaido 060-8638, Japan

E-mail: kaseron@med.hokudai.ac.jp

Key words: retinal hemangioma, laser photocoagulation, young girl, von Hippel Lindau disease retina, treatment options are basically retinal photocoagulation, cryopexy, and radiotherapy as well as photodynamic therapy (PDT) (4). In addition, vitrectomy and local tumor resection are important treatments, which can contribute to the preservation of patients' vision (5). However, when a tumor occurs at a juxtapapillary site, called a juxtapapillary retinal capillary hemangioma $(\mathrm{JRCH})$, treatment modalities may be more sight-threatening than for a non-growing tumor without complications (6), and a watch-and-wait strategy may initially be chosen. JRCH, one of the representative ocular manifestations in VHL disease (7), may cause various complications such as macular edema, retinal exudation, exudative retinal detachment, epiretinal membrane formation, and tractional retinal detachment (8). Two percent of patients with VHL disease and no retinal hemangiomas at the initial presentation eventually develop isolated JRCH (9). There is a risk of blindness caused by proliferative vitreoretinopathy and neovascular glaucoma (5) unless optimal treatments are conducted in a timely manner to treat active retinal tumors. Although ophthalmologists can conduct a number of primary treatments for adult patients with JRCH, little is known about the clinical course following retinal photocoagulation in $\mathrm{JRCH}$ of children. Indeed, it is likely that JRCH can occur in patients with VHL disease at an age of around 25 years, being earlier than in patients with sporadic tumors $(10,11)$. Herein, we report a young patient with suspected VHL disease presenting with JRCH.

\section{Case report}

A 6-year-old Japanese girl with suspected VHL disease was referred for the management of JRCH in her right eye. Her father died of renal cancer due to VHL disease at the age of 40. Her paternal grandfather had been diagnosed with VHL disease. Because it is highly penetrant, as $95 \%$ of patients with characterized mutations in the VHL gene manifest clinical disorders related to VHL disease by the age 60 years (12), we and the related clinical physicians have observed the child patient as suspected VHL disease. Visual acuity was 20/20 in both eyes. The fundus showed an orange-colored endophytic tumor located adjacent to the optic disc, measuring about $2.5 \mathrm{~mm}$, in the right eye (Fig. 1A). Fluorescein angiography (FA) revealed hyperfluorescence in the tumor in an early phase (Fig. 1B), where marked dye leakage was noted in a late 


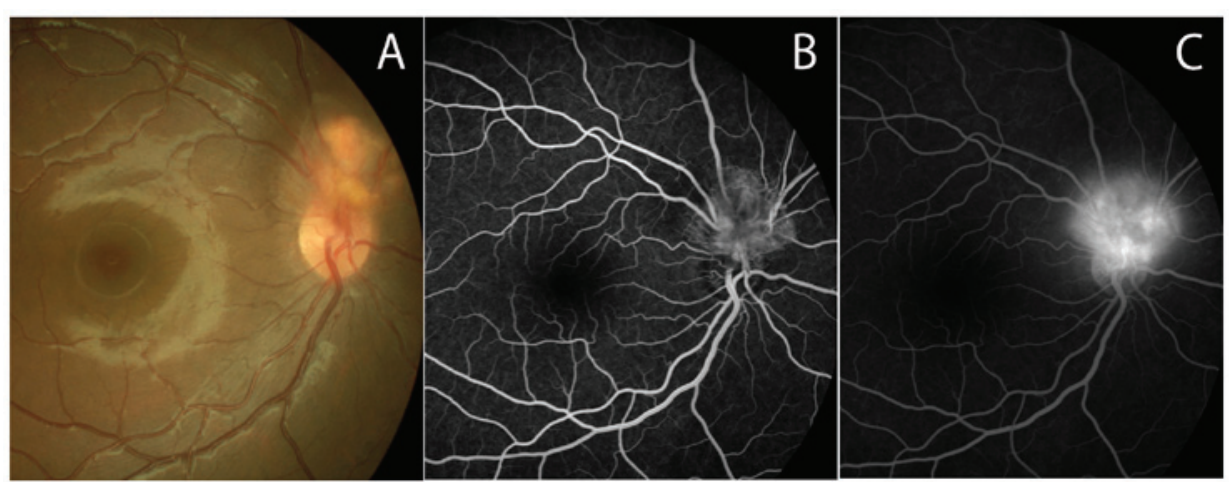

Figure 1. Fundus and fluorescein angiography in a 6-year-old girl. (A) Fundus shows an orange-colored endophytic tumor adjacent to the optic disc. (B) Fluorescein angiography shows hyperfluorescence in the tumor in an early phase, (C) where marked dye leakage may be noted in a late phase.

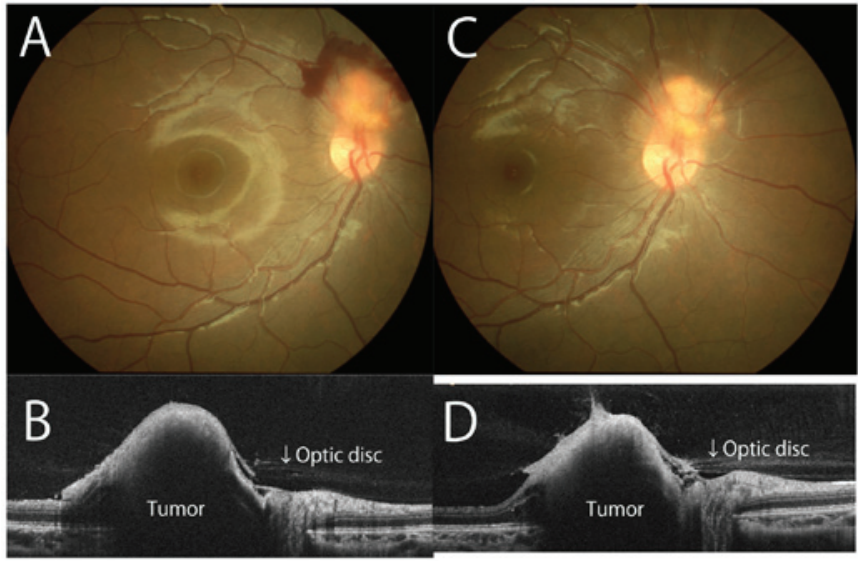

Figure 2. Fundus and OCT results in a 7-year-old girl 6 months after the initial presentation and 3 months after the direct laser photocoagulation. (A) Retinal hemorrhage is observed adjacent to the tumor, with a reddish appearance. (B) OCT shows an elevated lesion in the superior site of the optic disc. (C) Retinal hemorrhage diminished with no recurrence in the juxtapapillary region after photocoagulation. (D) An OCT imaging shows mild resolution of the elevated lesion with contraction of the vitreoretinal interface over the tumor. OCT, optical coherence tomography.

phase (Fig. 1C). Retinal hemorrhage, however, occurred around JRCH, with a reddish appearance, 7 months later (Fig. 2A). Optical coherence tomography (OCT) demonstrated an elevated lesion in the superior site of the optic disc (Fig. 2B). Since these results suggested potential tumor activity, primary treatments such as PDT, anti-vascular endothelial growth factor (VEGF) therapy, and laser photocoagulation were considered. Indeed, according to the previous report enrolling relatively large number of the patients, laser photocoagulation is one of the mainstays in treatments for retinal hemangioma (13). Moreover, this patient had undergone fundus examination using a super field non-contact lens several times. Therefore, direct photocoagulation of the tumor using the non-contact lens was considered the possible first-line treatment in this case. At the age of 7 years and 3 months, the patient underwent direct yellow laser ablation of the tumor using a super field non-contact lens (30 shots, $0.1 \mathrm{sec}$ in duration, $200 \mu \mathrm{m}$ spot size, and 0.1-0.20 W power) after informed consent was obtained from herself and her mother. The patient was orally asked to open her eyes during ablation. She did not suffer from ocular pain during treatment, and the ablation was safely completed. The hemorrhage gradually resolved one month later. There was no hemorrhage in the juxtapapillary region 6 months after photocoagulation (Fig. 2C). The tumor became paler than on initial presentation. OCT imaging showed mild resolution of the elevated lesion with contraction of the vitreoretinal interface over the tumor (Fig. 2D). Visual acuity remained 20/20 in both eyes. There was no recurrence of the hemorrhage and no enlargement of the tumor 9 months after the laser ablation.

\section{Discussion}

There are no established guidelines for the treatment of JRCH (14). On the other hand, eyes with de novo JRCH showed subsequent deterioration of visual acuity compared with either eyes without retinal involvement or eyes developing peripheral retinal hemangioma without JRCH (9). Moreover, multivariate logistic regression analyses verified the risk factors: a younger age at the baseline visit, and a younger age at the onset of ocular VHL disease (9). Therefore, the therapeutic indication should be carefully and strictly evaluated in a child with JRCH. Possible therapeutic options are PDT, laser photocoagulation, anti-VEGF therapy, radiotherapy, and cryopexy (6).

Since this case highlighted retinal hemorrhage surrounding JRCH with a reddish color and marked dye leakage on FA, treatments for the tumor were indicated because of the potential for progression. PDT might be chosen as the first-line treatment because it enables more selective vascular occlusion and reduces damage to the optic disc (4). However, PDT is a burdensome procedure for young patients. First of all, general anesthesia is desirable to start the treatment for children; however, the PDT procedure is quite complex under general anesthesia. Second, PDT basically requires laser ablation with contact lenses for $83 \mathrm{sec}$; however, wearing contact lenses under local topical eye drop anesthesia is impossible for children. In contrast, direct laser ablation is considered one of the established ocular treatments for JRCH in VHL disease (7). In fact, clinical outcomes following laser photocoagulation in children remain unknown, since this type of hemangioma usually occurs in older age groups, such as 25-year-old patients $(10,11)$. To the best of our knowledge, this report describes the youngest patient with VHL disease treated with direct laser ablation worldwide. Indeed, treatments for JRCH in VHL disease are challenging because there are potential 
risks of vision-related injury caused by the erroneous ablation of optic disc tissues and papillo-macular bundles. Therefore, optimal medical skills as well as the ability to establish favorable and trusting relationships with young patients should be key to overcome such complications. Therefore, the present results suggest that conventional laser photocoagulation should be considered as first-line treatment for JRCH.

It is debatable whether one session of laser photocoagulation is sufficient to achieve satisfactory inactivation and regression of the tumor, although this case was successfully treated with one session of direct laser ablation. Indeed, there will still be worries about optic disc injury, irreversible vision loss, and visual field defects on conducting multiple sessions of ablation. Therefore, careful observation was chosen after laser photocoagulation in this case. If the tumor grows, the coloration worsens, and retinal hemorrhage recurs, further laser ablation and intravitreal anti-VEGF injection combined with sub-Tenon triamcinolone acetonide injection (15) should be considered as second-line treatment.

\section{Acknowledgements}

Not applicable.

\section{Funding}

No funding was received.

\section{Availability of data and materials}

The datasets used and/or analyzed during the current study are available from the corresponding author on reasonable request.

\section{Authors' contributions}

SK and SI examined the patient, and SK wrote the medical records and wrote the manuscript. SK was responsible for surgical treatment. SI critically revised the paper. All authors read and approved the final manuscript.

\section{Ethics approval and consent to participate}

The Institutional Review Board at Hokkaido University (Sapporo, Japan) waived ethical approval for the present study, as it is a single case report.

\section{Patient consent for publication}

The patient provided consent for the publication of the case details and associated images.

\section{Competing interests}

The authors declare that they have no competing interests.

\section{References}

1. Lonser RR, Glenn GM, Walther M, Chew EY, Libutti SK, Linehan WM and Oldfield EH: von Hippel-Lindau disease. Lancet 361: 2059-2067, 2003.

2. Zou YU, Xu J and Zhang M: Long-term follow-up and clinical course of a rare case of von Hippel-Lindau disease: A case report and review of the literature. Oncol Lett 11: 3273-3278, 2016.

3. Bastos-Carvalho A and Damato B: Images in clinical medicine. Retinal hemangioblastoma in von Hippel-Lindau disease. N Engl J Med 363: 663, 2010.

4. Schmidt-Erfurth UM, Kusserow C, Barbazetto IA and Laqua H: Benefits and complications of photodynamic therapy of papillary capillary hemangiomas. Ophthalmology 109: 1256-1266, 2002.

5. Gaudric A, Krivosic V, Duguid G, Massin P, Giraud S and Richard S: Vitreoretinal surgery for severe retinal capillary hemangiomas in von hippel-lindau disease. Ophthalmology 118: 142-149, 2011.

6. Kase S and Ishida S: Retinal capillary hemangioma in von Hippel-Lindau disease: Current concept, diagnosis and managements. J Transl Med Epidemiol 2: 1010, 2014.

7. McCabe CM, Flynn HW Jr, Shields CL, Shields JA, Regillo CD, McDonald HR, Berrocal MH, Gass JD and Mieler WF: Juxtapapillary capillary hemangiomas. Clinical features and visual acuity outcomes. Ophthalmology 107: 2240-2248, 2000.

8. Magee MA, Kroll AJ, Lou PL and Ryan EA: Retinal capillary hemangiomas and von Hippel-Lindau disease. Semin Ophthalmol 21: 143-150, 2006.

9. Toy BC,Agrón E, Nigam D, Chew EY and Wong WT: Longitudinal analysis of retinal hemangioblastomatosis and visual function in ocular von Hippel-Lindau disease. Ophthalmology 119: 2622-2630, 2012.

10. Ridley M, Green J and Johnson G: Retinal angiomatosis: the ocular manifestations of von Hippel-Lindau disease. Can J Ophthalmol 21: 276-283, 1986.

11. Wittebol-Post D, Hes FJ and Lips CJ: The eye in von Hippel-Lindau disease. Long-term follow-up of screening and treatment: recommendations. J Intern Med 243: 555-561, 1998.

12. Maher ER, Yates JR, Harries R, Benjamin C, Harris R, Moore AT and Ferguson-Smith MA: Clinical features and natural history of von Hippel-Lindau disease. Q J Med 77: 1151-1163, 1990.

13. Singh AD, Nouri M, Shields CL, Shields JA and Perez N: Treatment of retinal capillary hemangioma. Ophthalmology 109: 1799-1806, 2002.

14. Saitta A, Nicolai M, Giovannini A and Mariotti C: Juxtapapillary retinal capillary hemangioma: new therapeutic strategies. Med Hypothesis Discov Innov Ophthalmol 3: 71-75, 2014.

15. Toyokawa N, Kimura H and Kuroda S: Juxtapapillary capillary hemangioma treated by intravitreal injection of bevacizumab combined with posterior subtenon injection of triamcinolone acetonide. Jpn J Ophthalmol 54: 168-170, 2010. 